\title{
A Cross-Layer Routing Protocol for Mobile Ad Hoc Networks Based On Minimum Interference Duration
}

\author{
Chao Gu, Qi Zhu \\ Jiangsu Key Lab of Wireless Communications, Key Lab on Wideband Wireless Communications and Sensor Network \\ Technology of Ministry of Education \\ Nanjing University of Posts and Telecommunications \\ Nanjing, China \\ yichao_gu@163.com, zhuqi@njupt.edu.cn
}

\begin{abstract}
Cross-layer design of routing protocol for mobile ad hoc networks (MANETs) has become a hot research topic in recent years. By integrating physical layer, MAC layer and network layer together for cross-layer design, a cross-layer routing protocol called MIR is proposed in this paper. Via predicting the duration of the interference imposed by the neighbors at every hop along the route, a new routing metric is presented which guarantees that the established routes will not break frequently while having the minimum interference. Simulation results show that MIR can significantly improve the network performance.
\end{abstract}

Keywords-ad hoc networks; cross-layer design; routing protocol; interference; NS

\section{INTRODUCTION}

Cross-layer design of routing protocol for MANETs has become a hot research topic in recent years. The main idea of cross-layer design is to take advantage of the correlations between the layers. That is, increasing the vertical communications among layers and decreasing the parallel communications among nodes to reduce the waste of resources and improve the performance of the network.

Existing analytical works on the cross-layer design of routing protocols based on interference in MANETs pay less attention to channel contention than to the number of the neighbor nodes [1, 2], which will bring two problems: (1) For a node in the network, only the neighbors that send or forward packets (i.e. the active neighbors) will interfere with it. So, it's inappropriate to use the number of neighbors to indicate the strength of the interference: (2)In most cases, nodes collect the neighbor information by periodically broadcasting the HELLO packets, which will not only increase the node workload but also deteriorate the network performance. We need

Because of the frequent change of network topology, the common defect of most routing protocols is that they cannot determine or predict the future network information according to the past and current network information. However, it is possible to solve this problem if we know the node mobility model. For this reason, studying the routing problems in MANETs based on specific node mobility models has become a hot topic recently $[3,4,5]$, and this paper is no exception.
In this paper, considering both the interference and the frequent link breakages in MANETs, we make use of the advantages of the cross-layer design to propose an interference-aware routing protocol called MIR (Minimum Interference Routing). By predicting the duration of the interference imposed by the neighbors at every hop along the route, a new routing metric is presented in MIR, which guaranteess that the established routes will not break frequently while having the minimum interference.

The rest of this paper is organized as follows: In section II, we define and compute the interference used in the paper. We propose the MIR protocol in section III and evaluate the performance of MIR in section IV. Finally, we give a summary of this paper in section $\mathrm{V}$.

\section{DEFINITION AND COMPUTATION OF THE INTERFERENCE}

\section{A. Definition of the Interference}

In order to describe interference objectively and accurately, we propose a more factual definition of interference. As depicted in Fig. 1, the zone covered by the solid line circle with the radius $R_{T X}$ is the transmission range while the zone covered by the doted line circle with the radius $R_{C S}$ is the carrier-sense range. Only the nodes within the transmission range could communicate with each other normally and only the nodes within the carrier-sense range could be sensed by each other. Here, we define the moments that two nodes move out of their transmission range and carrier-sense range as the link broken time $t_{\text {broken }}$

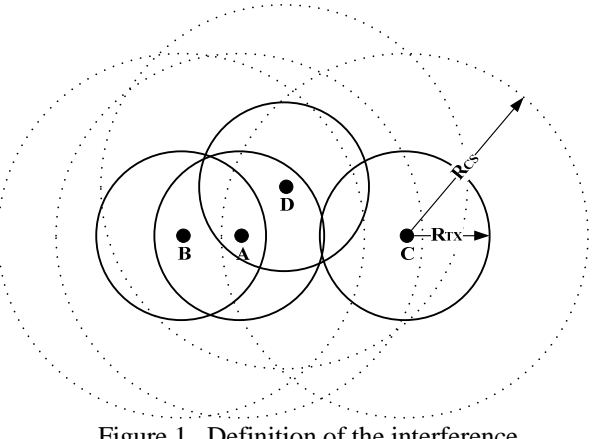

and node leave away time $t_{\text {leave_away }}$ respectively. 
Consider node $\mathrm{A}$ as the reference node and it is going to communicate with node B. According to IEEE 802.11 Distributed Coordination Function (DCF) [6], node C and node $\mathrm{D}$ will contend for the channel with node $\mathrm{A}$ as long as they are in the carrier-sense of node A. Channel contention makes node A defer sending the packets, which will make its buffer queue overflow more quickly and worsen the network performance ultimately.

Therefore, we define the interference imposed on node A by node $\mathrm{C}$ and node $\mathrm{D}$ as the duration they are in node A's carrier-sense range before link $\mathrm{AB}$ is broken. i.e.

$$
\text { Interference }_{A}=\sum_{i} \min \left(t_{\text {broken }}^{(A, B)}-t_{\text {current }}, T_{\text {duration }}^{i}\right)
$$

Where $i$ is the identification of node A's active neighbors, $t_{\text {current }}$ is the current time of the system and $T_{\text {duration }}^{i}$ is the duration that active neighbor node $i$ is in node A's carriersense range (i.e. $t_{\text {leave_away }}^{(A, i)}-t_{\text {current }}$ ).

In order to compute the interference, we must be able to predict $t_{\text {broken }}$ and $t_{\text {leave_away }}$.

\section{B. Computation of the interference}

So far, a lot of mobility models have been used in MANETs, including RWP model, random walk model, group mobility model etc. In this paper, we focus on RWP model. With this mobility model, each node selects a target location (i.e. waypoint) to move at a speed selected from a uniformly distributed interval $\left[V_{\min }, V_{\max }\right]$. Once the target is reached, the node pauses for a random time and then selects another target with another speed to move again.

Fig. 2 shows the prediction of $t_{\text {broken }}$ and $t_{\text {leave_away }}$. Assume that node $\mathrm{A}$ and node $\mathrm{B}$ move at velocities of $V_{\mathrm{A}}$ and $V_{B}$ respectively at a certain moment. If we consider node $\mathrm{A}$ as the reference node, then node $\mathrm{B}$ moves at a relative velocity of $V_{A B}$. According to the properties of RWP model [7], node B will keep this relative velocity in some distance. Since the distance between two nodes can be calculated by the received power and channel propagation model in the physical layer, node $\mathrm{A}$ can get the distance between itself and node $\mathrm{B}$ as long as node $\mathrm{B}$ sending packets (either control packets or data packets) within the carriersense range of node $\mathrm{A}$.

Suppose Node A has received packets from node B at moments $t_{0}, t_{1}$ and $t_{2}$, then we can get the distance between node $\mathrm{A}$ and node $\mathrm{B}$ at these moments. According to the law of cosines, we obtain the following equations:

$$
\left\{\begin{array}{l}
d_{t_{1}}{ }^{2}=d_{t_{0}}{ }^{2}+\left[V_{A B}\left(t_{1}-t_{0}\right)\right]^{2}-2 d_{t_{0}} V_{A B}\left(t_{1}-t_{0}\right) \cos \theta \\
d_{t_{2}}{ }^{2}=d_{t_{0}}{ }^{2}+\left[V_{A B}\left(t_{2}-t_{0}\right)\right]^{2}-2 d_{t_{0}} V_{A B}\left(t_{2}-t_{0}\right) \cos \theta \\
d_{t}{ }^{2}=d_{t_{0}}{ }^{2}+\left[V_{A B}\left(t-t_{0}\right)\right]^{2}-2 d_{t_{0}} V_{A B}\left(t-t_{0}\right) \cos \theta
\end{array}\right.
$$

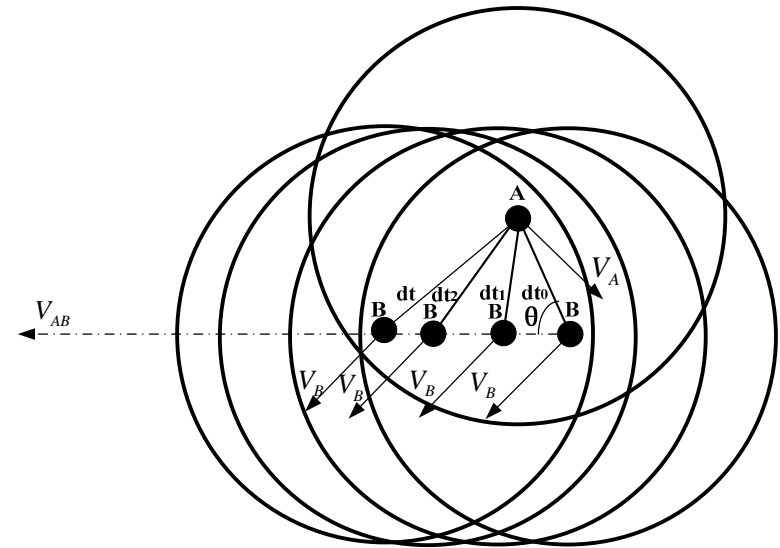

Figure 2. Prediction of $t_{\text {broken }}$ and $t_{\text {leave_away }}$

Then we can derive the distance between node $\mathrm{A}$ and node $\mathrm{B}$ at moment $t$

$$
d_{t}=\sqrt{a\left(t-t_{0}\right)^{2}+b\left(t-t_{0}\right)+c}
$$

where

$$
\left\{\begin{array}{l}
a=\frac{d_{t_{0}}{ }^{2}}{\left(t_{1}-t_{0}\right)\left(t_{2}-t_{0}\right)}-\frac{d_{t_{1}}{ }^{2}}{\left(t_{1}-t_{0}\right)\left(t_{2}-t_{1}\right)}+\frac{d_{t_{2}}{ }^{2}}{\left(t_{2}-t_{0}\right)\left(t_{2}-t_{1}\right)}=V_{A B}{ }^{2} \\
b=-\frac{\left(t_{1}+t_{2}-2 t_{0}\right) d_{t_{0}}{ }^{2}}{\left(t_{1}-t_{0}\right)\left(t_{2}-t_{0}\right)}+\frac{\left(t_{2}-t_{0}\right) d_{t_{1}}{ }^{2}}{\left(t_{1}-t_{0}\right)\left(t_{2}-t_{1}\right)}-\frac{\left(t_{1}-t_{0}\right) d_{t_{2}}{ }^{2}}{\left(t_{2}-t_{0}\right)\left(t_{2}-t_{1}\right)} \\
c=d_{t_{0}}^{2}
\end{array}\right.
$$

According to formula (3) and (4), we derive

$$
R_{T X}=\sqrt{a\left(t_{\text {broken }}-t_{0}\right)^{2}+b\left(t_{\text {broken }}-t_{0}\right)+c}
$$

Then we can get

$$
t_{\text {broken }}=\frac{\sqrt{b^{2}-4 a c+4 a R_{T X}^{2}}-b}{2 a}+t_{0}
$$

Similarly, we can get

$$
t_{\text {leave_away }}=\frac{\sqrt{b^{2}-4 a c+4 a R_{C S}^{2}}-b}{2 a}+t_{0}
$$

\section{The Minimum InTERference Cross-LAYER ROUTING PROTOCOL (MIR)}

Owing to the dynamic property of MANETs, metrics based on hop count may lead to some misjudgments, for the link may be easily broken or may be seriously interfered, which will worsen the network performance undoubtedly. Based on the acknowledgement, we propose the MIR protocol. Compared with other routing protocols, MIR first chooses the links that have long connectivity duration, and then it builds the least interfered route based on a new routing metric which takes interference and link connectivity duration together into account.

Here, we propose a simple and easy-to-implement method to guarantee long connectivity durations for the established routes. That is, as for node A and its next hop 
node $\mathrm{B}$ in a path, link $\mathrm{AB}$ will last for a long time if the link connectivity duration is greater than a certain threshold, i.e.

$$
t_{\text {broken }}^{(A, B)}-t_{\text {current }}>\text { Broken }_{\text {threshold }}
$$

where $t_{\text {broken }}^{(A, B)}$ can be obtained from formula (6) and $t_{\text {current }}$ is the current time of the system. Threshold (i.e. Broken $_{\text {threshold }}$ ) may be determined by the network conditions. For example, we may use a larger value if the network topology is fast-changing, and vice versa. The link will not be considered if the corresponding link connectivity duration is less than Broken $_{\text {threshold }}$.

Assert that Paths $=\left\{P_{1}, P_{2}, \ldots, P_{m}\right\}$ is a set contains $m$ paths from source node $\mathrm{S}$ to destination node $\mathrm{D}$. Set Hops $_{p_{t}}=\left\{h_{t}^{0}, h_{t}^{1}, \ldots, h_{t}^{l}\right\}$ represents the node sequences on path $P_{t}$. Based on the interference defined above, our routing metric is given by

$$
\text { Metric }=\min _{P_{i}}\left(\sum_{h_{i}^{j}} \frac{\frac{\text { Interference }_{j}}{\text { number }_{\text {interfer }}^{j}}}{t_{\text {broken }}^{(j-1, j)}-t_{\text {current }}}\right)
$$

where number ${ }_{\text {interfer }}^{j}$ is the total number of the interference nodes surrounding node $j, \frac{\text { Interference }_{j}}{\text { number }_{\text {interfer }}^{j}}$ is the mean duration of interference imposed on node $j$ and $t_{\text {broken }}^{(j-1, j)}-t_{\text {current }}$ is the connectivity duration of current communication link.

In a word, MIR will select the route with minimum interference and long connectivity duration.

\section{SIMULATION AND RESULT ANALYSIS}

\section{A. Simulation scenario and simulation parameters}

The simulation for evaluating MIR is carried out on NS2.33 [8]. The main simulation parameters are as follows. The scenario size is $1600 \times 1000 \mathrm{~m}^{2}$, the pause time and the max velocity are $0 s$ and $20 \mathrm{~m} / \mathrm{s}$; The channel bandwidth is $2 \mathrm{Mb} / \mathrm{s}, R_{T X}$ and $R_{C S}$ are set to $250 \mathrm{~m}$ and $550 \mathrm{~m}$ respectively; The number of nodes varies from 80 to 150 . We use constant bit rate (CBR) traffic and randomly choose 20 source-destination flows. The simulation time is 10 minutes and we set Broken $_{\text {threshold }}=10 \mathrm{~s}$.

We compare the performance MIR in terms of node density with the traditional AODV routing protocol by using the following performance metrics:

(1) Average end-to-end delay: It is defined as the average delay of a successful delivered CBR packet from the source node to the destination node.

(2) Packet loss ratio: It is defined as the ratio of the number of the lost data packets to the total number of data packets that are generated by the CBR sources.
(3) Throughput: It is defined as the ratio of the total size of the successful delivered CBR packets to the simulation time.

(4) Routing overhead: It is defined as the number of routing control packets that are needed to successfully deliver a CBR packet.

\section{B. Simulation result}

In order to evaluate the protocol with increasing node density, the max velocity of the nodes and the data rate are respectively set to $20 \mathrm{~m} / \mathrm{s}$ and 3 packets/s (i.e.12Kbps), while the other parameters are fixed, and the results are as follows.

Fig. 3 shows the relationship between the average endto-end delay and the node density. We can see that MIR reduces the delay by $28.5 \%$ compared with AODV on average, for it always selects the routes with minimum interference duration. The more the interference is, the longer the node will defer sending the packets, which will dramatically increase the delay.

The relationship between packet loss ratio and node density is shown in Fig. 4. Just as the same reason above, when node density increases, the growing interference results in high packet loss ratio. Owing to the unique characteristics of MIR, the packet loss ratio grows slower and decreases by $32.3 \%$ on average compared with that of AODV.

Fig. 5 shows the relationship between the throughput and the node density. The throughput of AODV is lower than that of MIR and decrease significantly with the sharp increase of the interference. On average, the throughput of MIR increases by $46.5 \%$ compared with that of AODV.

The relationship between the routing overhead and the node density is shown in Fig. 6. We can see that MIR has a better performance than AODV. The reason is that MIR ensures the established routes to last for a long time, which will greatly decrease the number of route reconstructions. Compared with AODV, the routing overhead of MIR decreases by $53.2 \%$ on average.

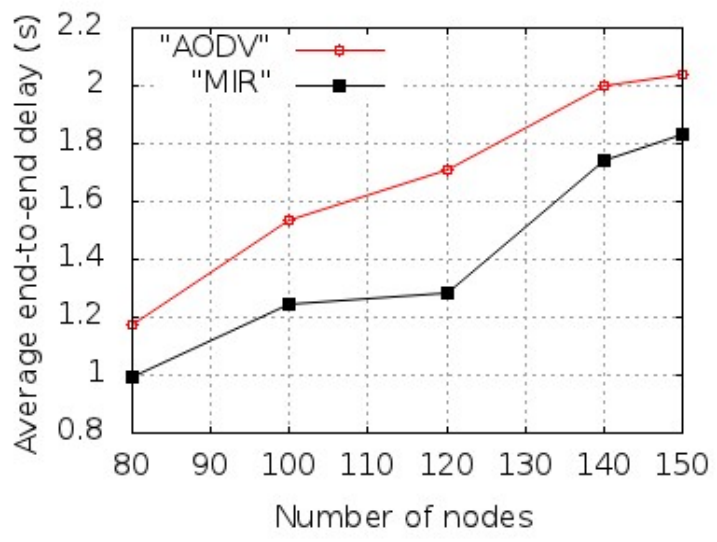

Figure. 3 Average end-to-end delay 

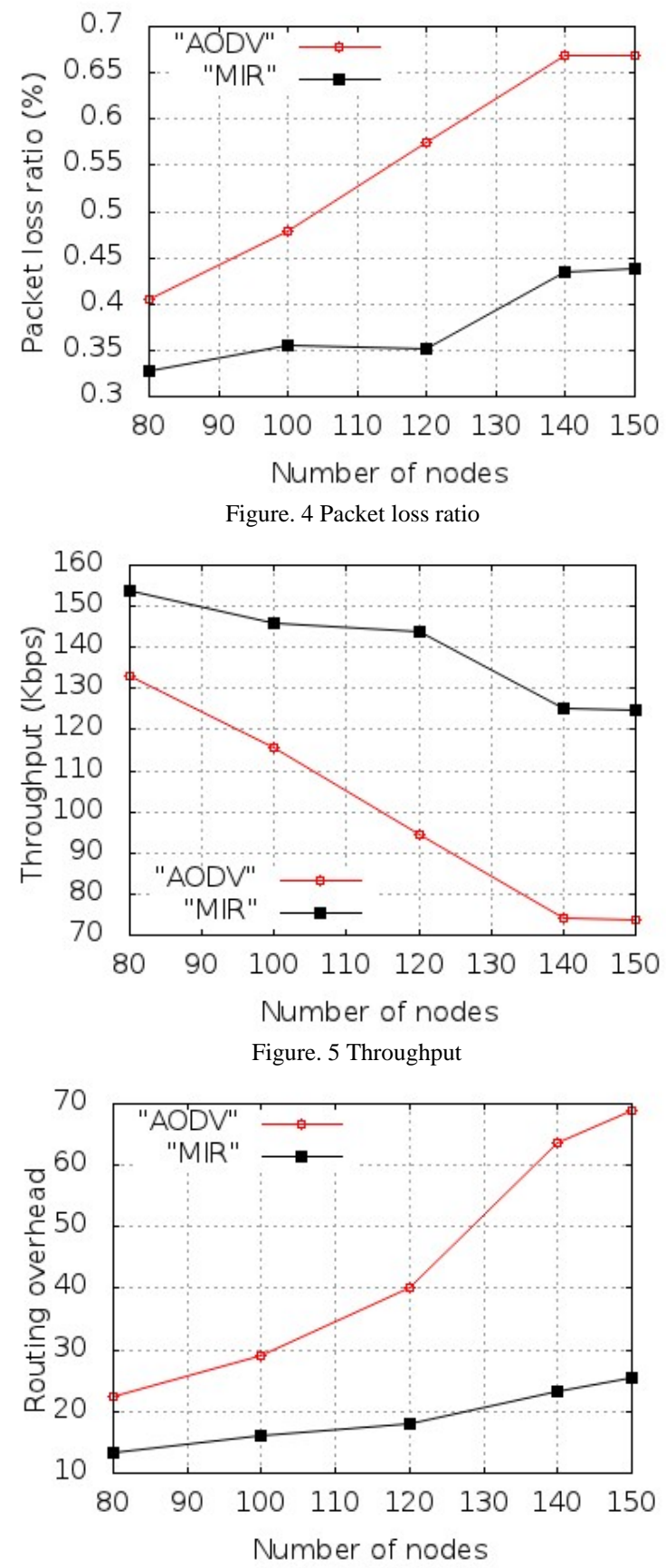

Figure. 6 Routing overhead

\section{CONCLUSION}

In this paper, we propose the MIR protocol for MANETs. This novel protocol makes full use of the advantages of the cross-layer design to improve the network performance. By using a new routing metric, the established routes will not break frequently while having the minimum interference. The interference imposed on the node can be more accurately determined by predicting the durations of the interference before the communication link is broken.
Simulation results show that MIR can significantly improve the network performance.

\section{ACKNOWLEDGMENT}

This work is supported by National Natural Science Foundation of China (61171094), National Science \& Technology Key Project (2011ZX03001-006-02, 2011ZX03005-004-03， 2012ZX03003011-005), National Basic Research Program of China (973 program: 2013CB329005) and Key Project of Jiangsu Provincial Natural Science Foundation (BK2011027).

\section{REFERENCES}

[1] B. C. Kim, J. Y. Lee, H. S. Lee, and J. S. Ma, “An Ad-Hoc Routing Protocol with Minimum Contention Time and Load Balancing," IEEE Global Telecommunications Conference, vol. 1, 2003, pp. 8185.

[2] X. M. Zhang, Q. Liu, D. Shi, Y. Z. Liu, and X. Yu, “An Average Link Interference-aware Routing Protocol for Mobile Ad hoc Networks," Third International Conference on IEEE Wireless and Mobile Communications, 2007.

[3] X. M. Zhang, F. F. Zou, E. B. Wang, and D. K. Sung, “ Exploring the dynamic nature of mobile nodes for predicting route lifetime in mobile adhoc networks," IEEE Transaction on Vehicular Technology, 2010, vol. 3, pp. 1567-1572.

[4] Q. Han, Y. Bai, L. Gong, and W. Wu, "Link availability predictionbased reliable routing for mobile adhoc networks," IET Communications, 2011, vol. 5, pp. 2291-2300.

[5] X. M. Zhang, E. B. Wang, J.J. Xia, and D.K. Sung, "An Estimated Distance-Based Routing Protocol for Mobile Ad hoc Networks," IEEE Transactions on Vehicular Technology, 2011, vol. 7, pp. 34733484.

[6] IEEE Standard for Wireless LAN Medium Access Control (MAC) and Physical Layer (PHY) specifications, IEEE 802.11b, 1999.

[7] C. Bettstetter, "Mobility modeling in wireless networks: Categorization, smooth movement, and border effects," ACM Mobile Computing and Communications Review, 2011, vol. 3, pp. 55-66.

[8] The Network Simulator - ns-2. http://www.isi.edu/nsnam/ns,2008. 